\title{
Robust Physical Alignment Models for Electron Tomography
}

Toby Sanders ${ }^{1}$, Micah Prange ${ }^{2}$, Peter Binev ${ }^{1}$, Cem Akatay $^{3}$, Ilke Arslan ${ }^{2}$

${ }^{1}$ University of South Carolina, Columbia, SC, 29208, USA

${ }^{2}$ Pacific Northwest National Laboratory, Richland, WA, 99352, USA

${ }^{3}$ UOP LLC, a Honeywell Company, 50 E. Algonquin Rd., 60016, Des Plaines, IL.

Tomography is a widely practiced technique for non-invasive 3-D imaging at all scales, with applications to many areas of science. In general, tomography involves three major phases: acquisition of 2-D projections of a sample, registration of the projections into a common coordinate system, and reconstruction of aligned data with a reconstruction algorithm. The acquisition methods and reconstruction algorithms have generated a great deal of interest due to the potential for improved resolution. On the other hand, the registration methods have not generated a great deal of research, and have typically used old hopeful models such as cross-correlation, or resorted to decorating the sample with gold particles for tracking. While one cannot improve the resolution capabilities with accurate alignment, inaccurate alignment or introduction of high density tracking particles can result in direct loss of resolution, making the alignment equally important.

Most recently, others have begun to use the physical motion of the center-of-mass of the sample as means for a more mathematically justifiable and noninvasive alignment technique [1,2]. These methods have proven useful, even capable of atomic resolution in cases [1]. However, even theoretically these methods can be shown to fall short of sufficient robustness in some simple settings, such as when the projected sample is not a fixed volume.

In our work, we develop more general alignment methods based on the center-of-mass, but allow for greater flexibility with our models by observing motions at many local scales, where the projected volume at these scales is in fact fixed. These observations allow us to continue to make use of the justifiable center-of-mass alignment methods and apply it in a much more general setting, making it a very robust approach.

In figures 1 and 2, reconstructions of an alumina particle are shown, where the data was aligned with our method and cross-correlation. In figure 1, the plot of the position of a local center-of-mass in the sample is given as a function of the projection angle, showing that the path of this mass follows a mathematically feasible trajectory, where cross-correlation fails to do so. Also in figure 1, a reconstruction of this region from the two alignments shows better results with our alignment. In figure 2, the full 3-D reconstructions are visualized with a volume rendering, in a color map that varies smoothly from black into red, and then to white. With cross-correlation, the red glow around the reconstructed particle is a result of blurring due to misalignment.

This research was funded by the Laboratory Directed Research and Development program at Pacific Northwest National Laboratory, under contract DE-AC05076RL01830. It was also funded in part by NSF grant DMS 1222390. 
[1] M.C. Scott, et al. in "Electron Tomography at 2.4 -angstrom resolution," Nature, 483, p.444447. (2012).

[2] T. Sanders, et al in "Physically Motivated Global Alignment Method for Electron Tomography," Adv. Chem. And Struct. Im. (2015).
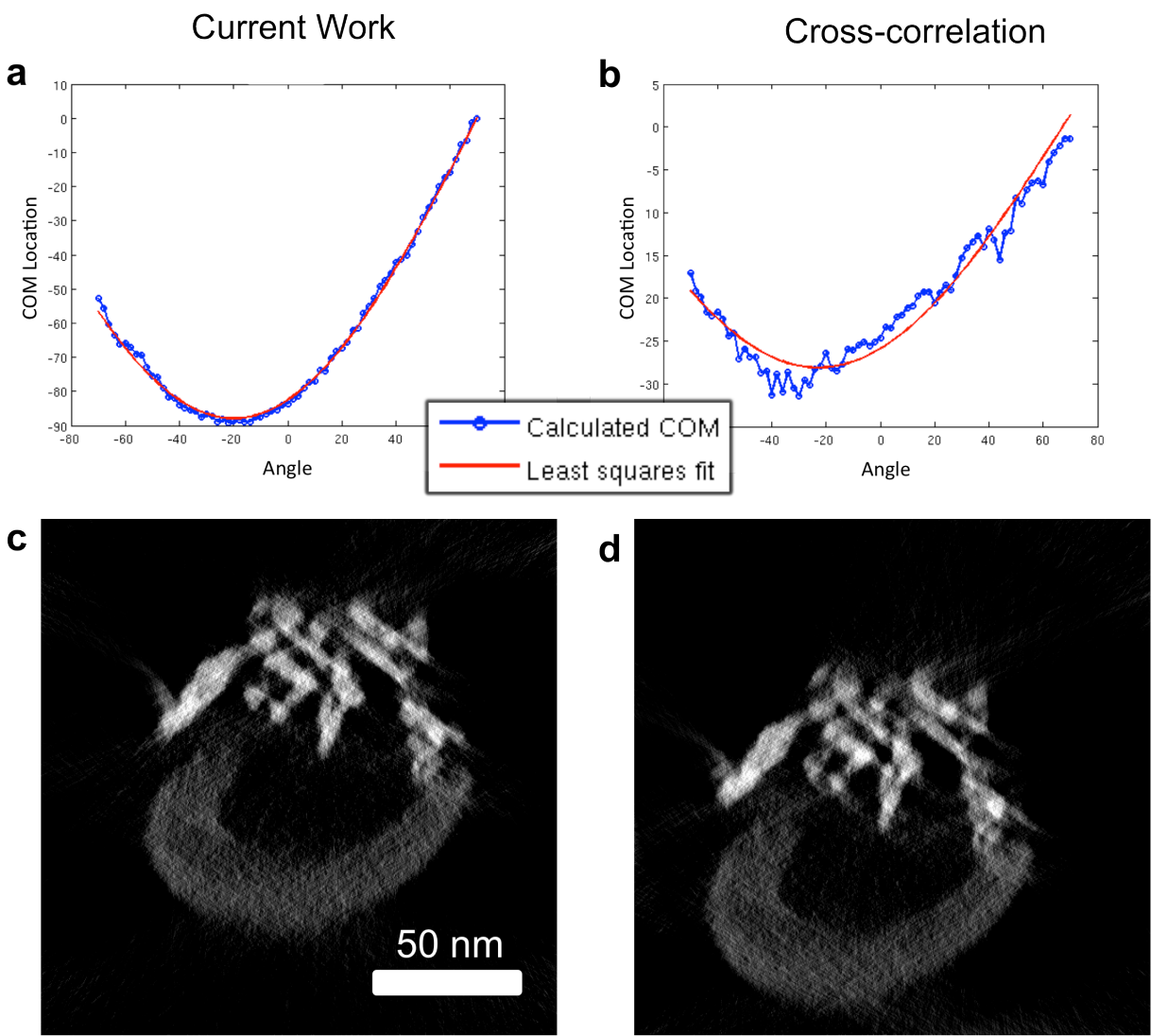

Figure 1: Left column: Results from our alignment. Right column: Results from cross-correlation alignment. a,b, Location of a local center-of-mass as a function of the projection angle. In blue is the calculated center-of-mass and in red is the best-fit curve for a feasible path of the center-of-mass. c,d, The resulting reconstruction of this local region.
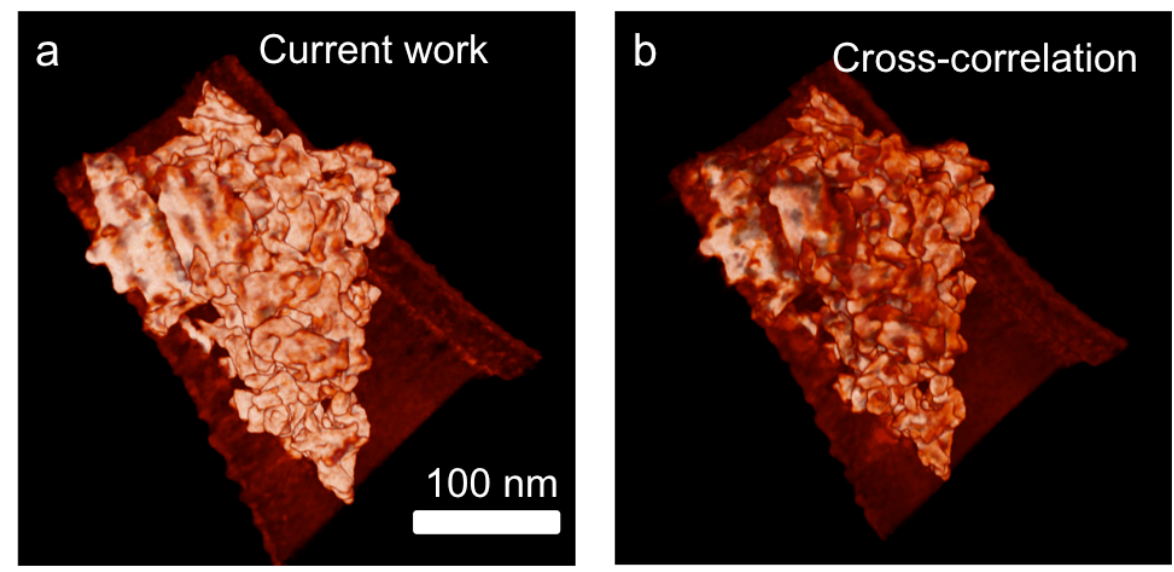

Figure 2: a, 3-D reconstruction from our alignment. b, 3-D reconstruction from cross-correlation. 